\title{
Effects of Quinidine on Short QT Syndrome Variant 2 in the Human Ventricle: A Modelling and Simulation Study
}

\author{
Cunjin Luo ${ }^{1,2}$, Kuanquan Wang ${ }^{1}$, Yang Liu ${ }^{1}$, Yong Xia ${ }^{3}$, Henggui Zhang ${ }^{1,2,4,5}$ \\ ${ }^{1}$ School of Computer Science and Technology, Harbin Institute of Technology, Harbin, China \\ ${ }^{2}$ Key Laboratory of Medical Electrophysiology, Ministry of Education, Collaborative Innovation \\ Centre for Prevention and Treatment of Cardiovascular Disease/Institute of Cardiovascular Research, \\ Southwest Medical University, Luzhou, China \\ ${ }^{3}$ School of Computer Science and Technology, Harbin Institute of Technology, Weihai, China \\ ${ }^{4}$ Space Institute of Southern China, Shenzhen, China \\ ${ }^{5}$ School of Physics and Astronomy, The University of Manchester, Manchester, United Kingdom
}

\begin{abstract}
The short QT syndrome (SQTS) is a rare cardiac disorder associated with an abnormally short QT interval and an increased risk of ventricular arrhythmias and sudden cardiac death (SCD). Gain-of-function mutation to potassium channels mediating the slow delayed rectifier current, $I_{K S}$, underlie KCNQ1-linked SQTS variant 2 (SQT2), in which treatment with sodium, calcium and potassium channel blocking class Ia antiarrhythmic agents has demonstrated some efficacy. This study used computational modelling and simulation to gain mechanistic insights into the actions the clinical drug, quinidine, in the setting of SQT2. The ten Tusscher et al. human ventricle model was modified to incorporate KCNQ1 V307L mutation-induced changes to $I_{K s}$ based on experimentally observed data: wild type (WT) and SQT2 mutant conditions were studied. Actions of quinidine were simulated by implementing a simple pore block theory to simulate the drug blocking effects on $I_{K r}, I_{K s}, I_{t o}, I_{N a}, I_{C a L}$, $I_{N a C a}$ and $I_{N a L}$, which were modelled using $I C_{50}$ and Hill coefficient. Cell models were incorporated into onedimensional (1D) model that considered the intrinsic electrical heterogeneity in the left ventricle. At a clinically therapeutic concentration of $10 \mu \mathrm{M}$ quinidine, the action potential duration (APD) was significantly increased, and the $Q T$ interval on the pseudo-ECG was prolonged. This study helps to better understand the underlying mechanisms of pharmacological therapy, and provides further evidence that quinidine is a suitable treatment for the SQT2 phenotype.
\end{abstract}

\section{Introduction}

The short QT syndrome (SQTS) is a cardiac disorder associated with abnormally short QT interval on the ECG, leading to increased risk of ventricular arrhythmias and sudden cardiac death (SCD) [1]. The SQTS is genetically heterogeneous, with a complex genotype-phenotype relationship. The SQT2 identified form of the SQTS was caused by a g919c substitution in KCNQ1 encoding the potassium channel. Functional studies of the KCNQ1 V307L mutation revealed a pronounced shift of the halfactivation potential and an acceleration of the activation kinetics leading to a gain of function in $I_{\mathrm{Ks}}$, which significantly reduces the QT interval of $290 \mathrm{~ms}$ (QTc, 302 ms) [2].

The current first-line treatment for SQTS patients is use of an implantable cardioverter-defibrillator (ICD) device, which protects against SCD [3]. However, the use of ICD device carries an increased risk of an inappropriate shock discharge by the ICD due to T-wave over-sensing. Furthermore, ICD does not restored the QT interval and, is not particularly suited to some pediatric patients, necessitating the pursuance of alternative, pharmacological approaches. Pharmacological therapy may be the primary modality to restore the physiological (normal) QT interval and protect against arrhythmias. At present, the accurate experimental models of SQT2 and in vitro pharmacological data on SQT2 patients are comparatively sparse. However, several studies have reported on the effectiveness of quinidine at restoring the QT interval in the setting of SQTS, as well as on the lack of effect of other anti-arrhythmic agents such as sotalol, ibutilide, and flecainide [4].

Our group recent studies [5,6] adopted computational a modelling and simulation approach to investigate the underlying mechanisms by which combined ion channel blocking actions of quinidine exert anti-arrhythmic effects in the setting of SQTS. Whereas, our group study [7] has previously used computer models to gain insights into QT interval shortening and pro-arrhythmic effects of 
SQT2 KCNQ1 V307L mutant $I_{\mathrm{K}}$ channels in human ventricles, significantly less is known about the pharmacological effects of anti-arrhythmic agents on SQT2. Although the effectiveness of quinidine has only been demonstrated in SQT1, there is a reason to believe that it may be useful in SQT2. Therefore, the present study was undertaken to gain mechanistic information regarding the actions of quinidine on human ventricular electrophysiology in the setting of V307L-linked SQT2.

\section{Methods}

\subsection{Model development}

The ten Tusscher et al. model [8] of the human ventricular action potential (AP) was used for simulations in this study, due to its extensive experimental validation and ability to reproduce complex behaviours such as reentrant excitation waves - a crucial requirement when simulating anti-arrhythmic effects of pharmacological agents on cardiac electrophysiology. Specifically, the single cell AP model can be modelled by using the following ordinary differential equation (ODE):

$$
\frac{d V_{m}}{d t}=-\frac{I_{i o n}+I_{\text {stim }}}{C_{m}}
$$

where $t$ is time, $C_{\mathrm{m}}$ is the cell membrane capacitance per unit surface area, $I_{\text {stim }}$ is the external stimulus current and $I_{\text {ion }}$ is the sum of the known transmembrane ionic currents. Particularly, the late sodium current $\left(I_{\mathrm{NaL}}\right)$ equation [9] was incorporated. The model code used in this study was downloaded from http://www-binf.bio.uu.nl/khwjtuss/. The cell model was paced with an amplitude of -52 $\mathrm{pA} / \mathrm{pF}$ for $1 \mathrm{~ms}$ and a basic cycle length (BCL) of $800 \mathrm{~ms}$.

Parameters in the equations for $I_{\mathrm{Ks}}$ were modified to incorporate experimental data of Bellocq et al. on SQT2 KCNQ1 V307L mutation-induced changes in $I_{\mathrm{Ks}}$ kinetics [2]. The $I_{\mathrm{Ks}}$ formulation is described by:

Original:

$$
\begin{gathered}
I_{K s}=G_{K s} x_{s}^{2}\left(V_{m}-E_{K}\right) \\
x_{s \infty}=\frac{1}{1+e^{\left(-5-V_{m}\right) / 14}} \\
\alpha_{x s}=\frac{1400}{\sqrt{1+e^{\left(5-V_{m}\right) / 6}}} \\
\beta_{x s}=\frac{1}{1+e^{\left(V_{m}-35\right) / 15}} \\
\tau_{x s}=\alpha_{x s} \beta_{x s}+80
\end{gathered}
$$

where $G_{\mathrm{Ks}}$ is maximal channel conductance, $x_{\mathrm{s}}$ is the activation variable, $V_{\mathrm{m}}$ is the transmembrane potential, $E_{\mathrm{K}}$ is the equilibrium potential, and $\tau_{x s}$ is the time constant.

Wild type (WT):

$$
\begin{gathered}
x_{s \infty}=\frac{1}{1+e^{\left(-5.9-V_{m}\right) / 17.4}} \\
\tau_{x s}=1 *\left(\alpha_{x s} \beta_{x s}+80\right)
\end{gathered}
$$

Heterozygous mutant expression (Het)

$$
\begin{gathered}
x_{s \infty}=\frac{1}{1+e^{\left(-20.62-V_{m}\right) / 10.96}} \\
\tau_{x s}=0.7 *\left(\alpha_{x s} \beta_{x s}+80\right)
\end{gathered}
$$

Homozygous mutant expression (Hom)

$$
\begin{gathered}
x_{s \infty}=\frac{1}{1+e^{\left(-24.05-V_{m}\right) / 16.09}} \\
\tau_{x s}=0.52 *\left(\alpha_{x s} \beta_{x s}+80\right)
\end{gathered}
$$

Homozygous mutant expression with reduced $I_{\mathrm{Ks}}$ (Homred)

$$
\begin{gathered}
x_{s \infty}=\frac{1}{1+e^{\left(-24.05-V_{m}\right) / 16.09}} \\
\tau_{x s}=0.32 *\left(\alpha_{x s} \beta_{x s}+80\right)
\end{gathered}
$$

A scaling factor ratio of 1.5: 1 in epicardial (EPI) $I_{\mathrm{Ks}}$ to mid-myocardial (MIDDLE) $I_{\mathrm{Ks}}$, was applied as reported in the study of Szabo et al. [10], in order to generate a high, symmetrical T-wave on the pseudo-ECG of SQTS.

\subsection{Drug modelling}

A simple pore block theory [11] was used for drug modelling. The effects of quinidine on $I_{\mathrm{Kr}}, I_{\mathrm{Ks}}, I_{\mathrm{to}}, I_{\mathrm{Na}}, I_{\mathrm{CaL}}$, $I_{\mathrm{NaCa}}$, and $I_{\mathrm{NaL}}$ were described using Hill coefficient $(\mathrm{nH})$ and half maximal inhibitory concentration $\left(\mathrm{IC}_{50}\right)$ values taken from the literature. The blocking potency of the drug on ionic currents is shown in Table 1. The therapeutic concentration of quinidine is $3.8 \sim 10.2 \mu \mathrm{M}$. Therefore, in this study $10 \mu \mathrm{M}$ was selected to simulate and predict the effects of quinidine on SQT2.

Table 1. Cardiac ion currents and conductivities (\% of original value) in the presence of $10 \mu \mathrm{M}$ quinidine.

\begin{tabular}{|l|l|l|l|l|}
\hline Current & $\mathrm{IC}_{50}$ & $\mathrm{nH}$ & Conductivity & Source \\
\hline$I_{\mathrm{Kr}}$ & 0.62 & 0.93 & $7 \%$ & {$[12]$} \\
\hline$I_{\mathrm{Ks}}$ & --- & --- & $45.2 \%$ & {$[13]$} \\
\hline$I_{\mathrm{to}}$ & 3.9 & 1 & $25.3 \%$ & {$[14]$} \\
\hline$I_{\mathrm{Na}}$ & 0.17 & 1 & $59.3 \%$ & {$[15]$} \\
\hline$I_{\mathrm{CaL}}$ & 14.9 & 1.1 & $61.2 \%$ & {$[16]$} \\
\hline$I_{\mathrm{NaCa}}$ & --- & --- & $86.5 \%$ & {$[16]$} \\
\hline$I_{\mathrm{NaL}}$ & 12 & 1 & $52.3 \%$ & {$[9]$} \\
\hline
\end{tabular}

\subsection{Tissue Simulation}

Initiation and conduction of action potentials (APs) in the multicellular tissue can be described by the following 
partial differential equation (PDE):

$$
C_{m} \frac{\partial V_{m}}{\partial t}=-\left(I_{i o n}+I_{s t i m}\right)+\nabla \cdot\left(D \nabla V_{m}\right)
$$

where $\nabla$ is the gradient operator defined within the tissue geometry, $D$ is the diffusion coefficient.

For one-dimensional (1D) simulations, a strand model was $15 \mathrm{~mm}$ and employed a spatial resolution of $0.15 \mathrm{~mm}$, close to the ventricular cell length of $80 \sim 150 \mu \mathrm{m}$. The strand had 25 nodes for ENDO, 35 nodes for MIDDLE and 40 nodes for EPI cells. The total length and proportion for each sub-region reliably reproduced a positive T wave on the pseudo-ECG. $D$ was set at 0.0008 $\mathrm{cm}^{2} / \mathrm{ms}$, which promoted a conduction velocity $(\mathrm{CV})$ of $52 \mathrm{~cm} / \mathrm{s}$, close to the experimental CV of $\sim 50 \mathrm{~cm} / \mathrm{s}$.

A pseudo-ECG was calculated by the following expression [17]:

$$
\phi_{e}\left(x^{\prime}\right)=\frac{\alpha^{2}}{4} \int\left(-\nabla V_{m}\right) \cdot \nabla\left(\frac{1}{r}\right) d x
$$

where $\alpha$ is the radius of the 1D strand, $d x$ is the spatial resolution, $r$ is the Euclidean distance form a strand point $x$ to the electrode point $x$. The virtual electrode was placed at a position $2.0 \mathrm{~cm}$ away from the EPI end of the strand.

\section{Results}

Figure 1 shows the simulated ENDO, MIDDLE and EPI cell action potentials and $I_{\mathrm{Ks}}$ profile with the modified $I_{\mathrm{Ks}}$ equations. These plots show cell action potential $V$ $(\mathrm{mV})$ versus time $t(\mathrm{~ms})$, stimulated by a $1.25 \mathrm{~Hz}$ frequency. The $I_{\mathrm{Ks}}$ in the WT condition increased progressively, however, in the SQT2 conditions, $I_{\mathrm{Ks}}$ increased more rapidly, and reached a higher peak amplitude, leading to the shortening of the action potential duration. This results indicate that the modified model can capture the features of the WT and mutant $I_{\mathrm{Ks}}$ channel dynamics, and suggest that it can be used to predict the effects of quinidine on SQT2.

The effects of $10 \mu \mathrm{M}$ quinidine on the cell action potentials are shown in Figure 2. The results indicate that quinidine markedly decreased the action potential amplitude and prolonged the action potential duration in SQT2 conditions. A pseudo-ECG was simulated by using a 1D strand model as shown in Figure 3. The QT interval on the ECG was prolonged in SQT2 conditions by the application of $10 \mu \mathrm{M}$ quinidine. Especially, the QT interval in the simulated dose of quinidine under the Het condition was prolonged to the normal physiological range of QT interval between 363 and $421 \mathrm{~ms}$.

\section{Conclusion}

In the present study, we have showed that $10 \mu \mathrm{M}$ quinidine produced a therapeutic effect on ventricular electrophysiology in SQT2 conditions. This study provides new evidence that quinidine may be a potential pharmacological agent for treating the KCNQ1-linked SQT2 patients.
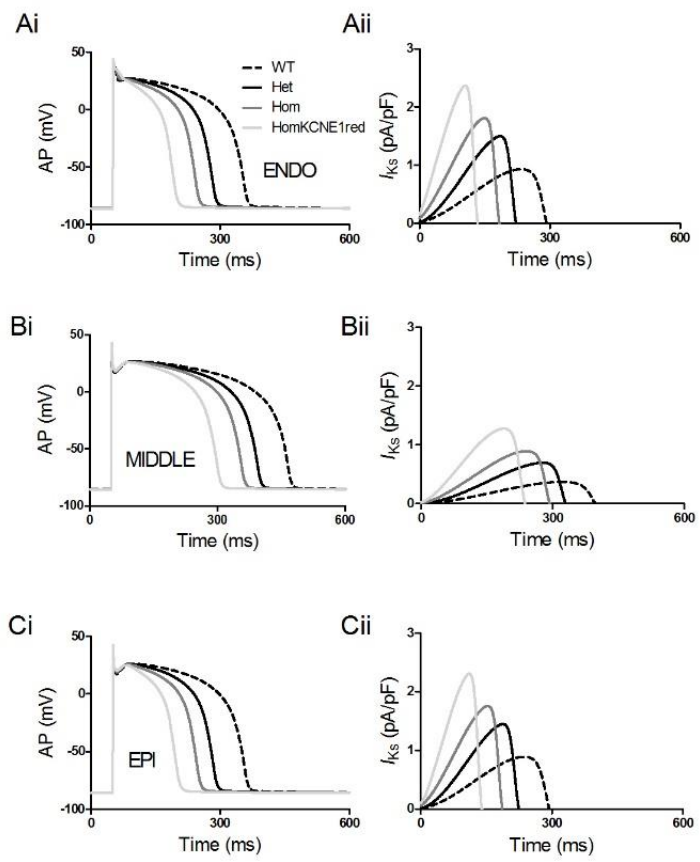

Figure 1. Simulations of ENDO, MIDDLE and EPI cell action potentials together with the corresponding time course of IKs in WT and SQT2 conditions.
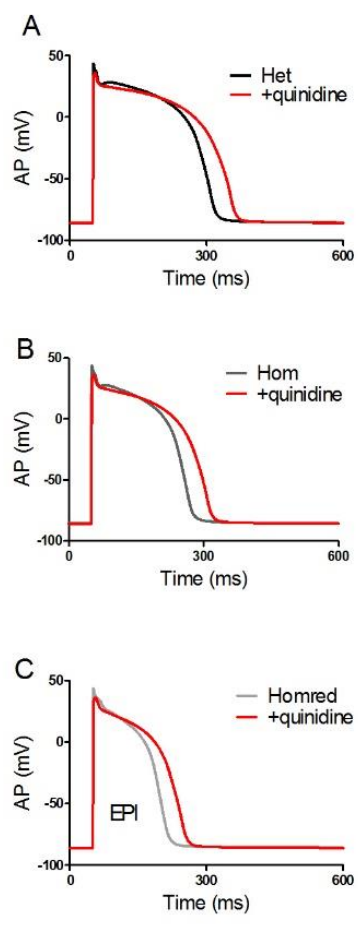

Figure 2. The pharmacological effects of quinidine on cell action potentials in SQT2 conditions. 


\section{Acknowledgements}

The authors thank Dr. Ismail Adeniran for useful discussions. This study was supported by the China Scholarship Council (CSC), the National Science Foundation of China (NSFC) under Grants No. 61571165 and No. 61572152, and Shandong Province Natural Science Foundation under Grant No. ZR2015FM028.
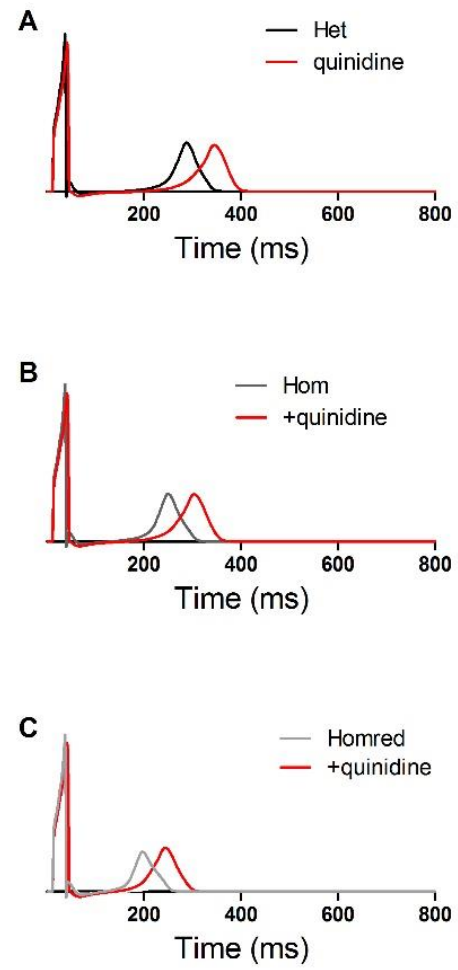

Figure 3. The pharmacological effects of quinidine on pseudo-ECGs in SQT2 conditions.

\section{References}

1. Gussak I, Brugada P, Brugada J, Wright RS, Kopecky SL, et al. (2000) Idiopathic short QT interval: a new clinical syndrome? Cardiology 94: 99-102.

2. Bellocq C, van Ginneken AC, Bezzina CR, Alders M, Escande D, et al. (2004) Mutation in the KCNQ1 gene leading to the short QT-interval syndrome. Circulation 109: 2394-2397.

3. Schimpf R, Wolpert C, Bianchi F, Giustetto C, Gaita F, et al. (2003) Congenital short QT syndrome and implantable cardioverter defibrillator treatment: inherent risk for inappropriate shock delivery. J Cardiovasc Electrophysiol 14: 1273-1277.

4. Gaita F, Giustetto C, Bianchi F, Schimpf R, Haissaguerre M, et al. (2004) Short QT syndrome: pharmacological treatment. J Am Coll Cardiol 43: 1494-1499.

5. Luo C, Wang K, Zhang H (2017) Modelling the effects of quinidine, disopyramide, and E-4031 on short QT syndrome variant 3 in the human ventricles. Physiol Meas 38: 1859-1873.

6. Luo C, Wang K, Zhang H (2017) In silico assessment of the effects of quinidine, disopyramide and E-4031 on short QT syndrome variant 1 in the human ventricles. PLoS One 12: e0179515.

7. Zhang H, Kharche S, Holden AV, Hancox JC (2008) Repolarisation and vulnerability to re-entry in the human heart with short QT syndrome arising from KCNQ1 mutation--a simulation study. Prog Biophys Mol Biol 96: 112-131.

8. ten Tusscher KH, Panfilov AV (2006) Alternans and spiral breakup in a human ventricular tissue model. Am J Physiol Heart Circ Physiol 291: H1088-1100.

9. O'Hara T, Virag L, Varro A, Rudy Y (2011) Simulation of the undiseased human cardiac ventricular action potential: model formulation and experimental validation. PLoS Comput Biol 7: e1002061.

10. Szabo G, Szentandrassy N, Biro T, Toth BI, Czifra G, et al. (2005) Asymmetrical distribution of ion channels in canine and human left-ventricular wall: epicardium versus midmyocardium. Pflugers Arch 450: 307-316.

11. Brennan T, Fink M, Rodriguez B (2009) Multiscale modelling of drug-induced effects on cardiac electrophysiological activity. Eur J Pharm Sci 36: $62-$ 77.

12. McPate MJ, Duncan RS, Hancox JC, Witchel HJ (2008) Pharmacology of the short QT syndrome N588KhERG K+ channel mutation: differential impact on selected class I and class III antiarrhythmic drugs. Br J Pharmacol 155: 957-966.

13. Yang T, Kanki H, Zhang W, Roden DM (2009) Probing the mechanisms underlying modulation of quinidine sensitivity to cardiac $\mathrm{I}(\mathrm{Ks})$ block by protein kinase Amediated I(Ks) phosphorylation. Br J Pharmacol 157: 952-961.

14. Slawsky MT, Castle NA (1994) K+ channel blocking actions of flecainide compared with those of propafenone and quinidine in adult rat ventricular myocytes. J Pharmacol Exp Ther 269: 66-74.

15. Koumi S, Sato R, Hayakawa H, Okumura H (1991) Quinidine blocks cardiac sodium current after removal of the fast inactivation process with chloramine-T. J Mol Cell Cardiol 23: 427-438.

16. Zhang YH, Hancox JC (2002) Mode-dependent inhibition by quinidine of $\mathrm{Na}+\mathrm{Ca} 2+$ exchanger current from guinea-pig isolated ventricular myocytes. Clin Exp Pharmacol Physiol 29: 777-781.

17. Gima K, Rudy Y (2002) Ionic current basis of electrocardiographic waveforms: a model study. Circ Res 90: 889-896.

Address for correspondence.

Name: Cunjin Luo

Full postal address: Room 306, Integrated Laboratory Building, School of Computer Science and Technology, Harbin Institute of Technology, Xidazhi Street, Nangang District, Harbin, 150001, China.

E-mail address: cunjin.luo@yahoo.co.uk 